\title{
Relative extended haplotype homozygosity signals across breeds reveal dairy and beef specific signatures of selection
}

\author{
Lorenzo Bomba ${ }^{* *}$, Ezequiel L Nicolazzi ${ }^{2+}$, Marco Milanesi ${ }^{1}$, Riccardo Negrini ${ }^{3}$, Giordano Mancini ${ }^{4}$, Filippo Biscarini ${ }^{2}$, \\ Alessandra Stella ${ }^{2,5}$, Alessio Valentini ${ }^{6}$ and Paolo Ajmone-Marsan ${ }^{1}$
}

\begin{abstract}
Background: A number of methods are available to scan a genome for selection signatures by evaluating patterns of diversity within and between breeds. Among these, "extended haplotype homozygosity" (EHH) is a reliable approach to detect genome regions under recent selective pressure. The objective of this study was to use this approach to identify regions that are under recent positive selection and shared by the most representative Italian dairy and beef cattle breeds.
\end{abstract}

Results: A total of 3220 animals from Italian Holstein (2179), Italian Brown (775), Simmental (493), Marchigiana (485) and Piedmontese (379) breeds were genotyped with the Illumina BovineSNP50 BeadChip v.1. After standard quality control procedures, genotypes were phased and core haplotypes were identified. The decay of linkage disequilibrium (LD) for each core haplotype was assessed by measuring the $\mathrm{EHH}$. Since accurate estimates of local recombination rates were not available, relative EHH ( $\mathrm{rEHH})$ was calculated for each core haplotype. Genomic regions that carry frequent core haplotypes and with significant $\mathrm{rEHH}$ values were considered as candidates for recent positive selection. Candidate regions were aligned across to identify signals shared by dairy or beef cattle breeds. Overall, 82 and 87 common regions were detected among dairy and beef cattle breeds, respectively. Bioinformatic analysis identified 244 and 232 genes in these common genomic regions. Gene annotation and pathway analysis showed that these genes are involved in molecular functions that are biologically related to milk or meat production.

Conclusions: Our results suggest that a multi-breed approach can lead to the identification of genomic signatures in breeds of cattle that are selected for the same production goal and thus to the localisation of genomic regions of interest in dairy and beef production.

\section{Background}

Advances in genomic technologies and the availability of single nucleotide polymorphism (SNP) markers have enabled genome-wide studies of the effect of selection in cattle $[1,2]$. Selection signals that result from environmental or anthropogenic pressures help us understand the processes that have led to breed formation. These studies are usually conducted with a "top-down" approach [3], from genotype to phenotype, whereby genomic data are statistically analysed to detect traces/marks/signs of directional selection. In analyses that aim at identifying

\footnotetext{
*Correspondence: Iorenzo.bomba@unicatt.it

${ }^{\dagger}$ Equal contributors

'Istituto di Zootecnica, UCSC, via Emilia Parmense 84, Piacenza 29122, Italy

Full list of author information is available at the end of the article
}

selection signatures, the phenotype is considered in its broadest sense: breed, production aptitude or even adaptation to a specific environment. This approach holds the potential to investigate traits that are very expensive, difficult and sometimes impossible to study with classical GWAS (genome-wide association study) approaches, such as tolerance to extreme climates or various feeding and husbandry systems, resilience to diseases, etc. Therefore, results from these studies are complementary to those from GWAS for investigating the molecular mechanisms that underlie important biological processes [4]. Many methods have been proposed to scan for selection signatures at the genomic level [5] by analysing either withinor across-breeds patterns of diversity by comparing allele or haplotype frequencies and sizes, alleles that are 
segregating or fixed in populations, and to preferentially detect recent or ancient selection events [5-7]. Different methods have different sensitivities and robustness, e.g. they may be influenced to a different extent by marker ascertainment bias and uneven distribution of recombination hotspots along the genome.

Extended haplotype homozygosity (EHH), a method that identifies long-range haplotypes, was developed by Sabeti et al. [8] for applications in human genetics and has been applied to many animal species, including cattle $[2,9,10]$. Under a neutral evolution model, changes in allele frequencies are assumed to be driven only by genetic drift. In this scenario, a new variant will require many generations to reach a high frequency in the population, and the surrounding linkage disequilibrium (LD) will decay due to recombination events [11]. Conversely, in the case of positive selection, a rapid rise in frequency of a beneficial mutation in a relatively few generations will preserve the original haplotype structure (core haplotype), since the number of recombination events would be limited. Therefore, based on EHH, a positive selection signature is defined as a region characterized by strong and long-range LD and having an allele within an uncommonly high frequency haplotype.

The EHH method detects genomic regions that are candidates for having undergone recent selection and, unlike integrated haplotype score (iHS) [12], does not require the definition of ancestral alleles. In addition, it is suited to the analysis of SNP data, because it is less sensitive to ascertainment bias than other methods [4]. However, EHH is likely to generate a large number of false positive and false negative results, due to heterogeneous recombination rates along the genome [2]. An additional drawback that is shared by all selection signature methods, including $\mathrm{EHH}$, is the challenge of robust inference, e.g. the ability to distinguish between true and spurious signals [13].

To partially account for these limitations, Sabeti et al. [8] developed the relative extended haplotype homozygosity (rEHH) method, which applies an empirical approach to assess the significance of signals. The rEHH of a core haplotype (i.e. short region in strong LD along the genome) is compared with the EHH value of other haplotypes at the same locus of the core haplotype, using these as a control for local variation in recombination rates. Therefore, it only identifies genomic regions, which carry variants under selection that are still segregating in the population. Although EHH and rEHH methods were developed for human population studies, they have been successfully applied to livestock species, such as pig [14] and cattle [2].

After domestication, which occurred about 10000 years ago in the fertile crescent, taurine cattle colonized Europe and Africa and were selected to satisfy different human needs [15]. During the last century, anthropogenic pressure has led to the formation of hundreds of specialized breeds that are adapted to different environmental conditions and linked to local traditions, constituting a gene pool relevant for conservation [16]. Some of these breeds have experienced strong artificial selection for dairy, beef, or both production specializations [17]. The present study uses the rEHH method to identify signals of recent directional selection in dairy and beef production, using five Italian dairy, beef and dual purpose cattle breeds. We focused on significant core haplotypes that are shared by breeds selected for the same production type. Finally, we identified positional candidate genes within the genomic regions under selection and investigated their biological role.

\section{Methods}

\section{Animals sampled and genotyping}

A total of 4311 bulls from five Italian dairy, beef, and dual purpose breeds were genotyped with the Illumina BovineSNP50 BeadChip v.1 (Illumina, San Diego, CA), by combining genotyping efforts of two Italian projects ("SelMol" and "Prozoo"). The dataset included 101 replicates and 773 sire-son pairs, used for downstream quality checking of the data produced. The genotypes of 2954 dairy (2179 Italian Holstein and 775 Italian Brown), 864 beef (485 Marchigiana and 379 Piedmontese) and 493 dual purpose (Italian Simmental) bulls were available. Data quality control (QC) was performed in two steps: first on animals, independently in each breed, by applying the same filters and thresholds, and then on markers, across all individuals in the dataset. The first step excluded individuals with unexpectedly high $(\geq 0.2 \%)$ Mendelian errors for father-son pairs and individuals with low call rates $(\leq 95 \%)$. The second step excluded: (i) SNPs with more than $2.5 \%$ missing values in the whole dataset or completely missing in one breed; (ii) SNPs with a minor allele frequency less than 5\%; and (iii) SNPs that were located on the sex chromosomes or for which chromosome assignment or physical position was lacking.

\section{Estimation of $\mathrm{rEHH}$}

Haplotypes were obtained by fastPHASE using the default options [18], and were run by breed and chromosome for each breed. Pedigree information for all bulls was provided by breed associations, and were used to filter out direct relatives (in father-son pairs, the son was maintained in the dataset and the father removed) and over-represented families (a maximum of five randomly chosen individuals per half-sib family was allowed). The final dataset containing these "less-related" animals is referred to as the "non-redundant" dataset and was used to calculate the within-breed pair-wise LD. The $\mathrm{r}^{2}$ 
statistic for all pairs of markers was calculated using PLINK v.1.0.7 [19]. The decay of LD was estimated by averaging $\mathrm{r}^{2}$ values as a function of marker distance, up to $1 \mathrm{Mb}$.

To test if population structure influenced $\mathrm{rEHH}$ detection, we repeated the whole Italian Holstein dataset analysis (i.e. the "redundant" dataset comprising fatherson pairs and all available half-sibs per family) and focused on genes or gene clusters that are well known to be under recent selection in cattle (i.e. "control regions"). In particular, we focused on the casein gene cluster, the polled locus and on two coat colour genes (MC1R and KIT $[2,13])$.

EHH and rEHH were calculated by Sweep v.1.1 [8]. Some default program settings had to be modified to adapt the analysis to the bovine genome. Specifically, local recombination rates between SNPs were approximated to $1 \mathrm{cM}$ per $\mathrm{Mb}$. EHH and $\mathrm{rEHH}$ calculations were performed by breed and chromosome, using automatic haplotype core selection with default options, i.e. considering the longest non-overlapping haplotype cores and limiting haplotype cores to at least three and no more than 20 SNPs, as in Qanbari et al. [2]. To set an (empirical) $\mathrm{rEHH}$ significance threshold, we first split $\mathrm{rEHH}$ values into 20 bins with a frequency range of $5 \%$ each, then log-transformed within-bin values to achieve normality, and finally considered significant core haplotypes with a $p$-value less than 0.05 . Although EHH and rEHH values were obtained for all core haplotypes, only those with a frequency greater than $25 \%$ were retained for further analyses.

\section{Breed grouping according to production type}

Regions under putative selection for dairy and beef production were identified from significant core haplotypes that shared one or more SNPs in at least two breeds with the same production type. The dual purpose Italian Simmental was included in both dairy (Italian Holstein and Italian Brown) and beef breeds (Piedmontese and Marchigiana), since this breed potentially possesses haplotypes that have been selected for both production types. All downstream analyses were performed separately for the dairy and beef breeds.

\section{Detection and annotation of candidate genes}

The genomic coordinates (in bp) of the regions shared by dairy or by beef breeds were used as inputs to retrieve gene information and annotation from the Biomart web interface (http://www.ensembl.org/biomart/ martview). The resulting gene set was then used as input for a canonical pathway analysis by examining the functional relationships among the resulting genes using Ingenuity Pathway Analysis tool version 8.0 (IPA; Ingenuity Systems, Inc, Redwood City, CA; http://www.ingenuity. com), coupled with a detailed examination of the literature. IPA operates with a proprietary knowledge database, providing pathway analysis for several species, including cattle. For IPA analysis, Fisher's exact test following a Benjamini and Hochberg correction for multiple-testing was used to estimate the significance of each biological function.

\section{Results}

\section{Quality control of the dataset}

Reproducibility observed from analysis of 101 replicates in the whole dataset was greater than $99.8 \%$. After the two quality control steps, 105 individuals and 9730 SNPs were removed. After phasing, 1292 additional individuals were removed to reduce the large number of sib-families present in the redundant dataset. The final dataset contained 44 271 SNPs and 1132, 514, 393, 410 and 364 individuals from Italian Holstein, Italian Brown, Italian Simmental, Marchigiana and Piedmontese breeds, respectively (Table 1).

\section{Assessing the effect of population structure in control regions}

Comparison of $\mathrm{rEHH}$ at the four selected control regions (casein gene cluster, polled locus, MC1R and KIT genes) based on analysis of the redundant versus nonredundant datasets indicated that population structure (redundant vs. non-redundant datasets) has an influence on the detection of selection sweeps. In fact, in the redundant dataset, Sweep v1.1. detected only one significant core haplotype at the casein gene cluster, while in the non-redundant dataset significant haplotypes were also found at the polled locus and at the MC1R gene. No significant signal was detected at the KIT gene in either dataset (Table 2). Plots of EHH vs. distance for the two most frequent haplotypes around the genes with significant $\mathrm{rEHH}$ (casein gene cluster, polled locus and MC1R) are in Figure 1. All subsequent analyses were conducted on the non-redundant dataset which, although of smaller size, proved more informative than the entire dataset.

\section{Detection of selection signatures}

In total, $17363,17801,14837,13814$, and 12747 core haplotypes with a frequency greater than $25 \%$ were detected in the Italian Holstein, Italian Brown, Italian Simmental, Marchigiana and Piedmontese breeds, respectively. The genome-wide distributions of $p$ values for $\mathrm{rEHH}$ for each breed are in Additional file 1 [See Additional file 1: Figures S1, S2, S3, S4 and S5]. In total $838,866,740,692$ and 613 core haplotypes were found to be significant ( $p$ values $\leq 0.05$ ) in the aforementioned breeds. Table 3 shows the distribution of total and significant core haplotypes per chromosome and breed. 
Table 1 Number of animals genotyped before and after quality control

\begin{tabular}{llllll}
\hline Breed & Total genotyped & ED- 5\% misAN & ED-REPL & ED- MEND & Cleaned \\
\hline HOL & 2179 & 40 & 31 & 5 & 2093 \\
BRW & 775 & 6 & 16 & 4 & 749 \\
SIM & 493 & 6 & 6 & 2 & 479 \\
MAR & 485 & 37 & 38 & - & 410 \\
PIE & 379 & 5 & 10 & - & 364
\end{tabular}

Total number of animals genotyped and number of animals removed after quality control analysis; $\mathrm{HOL}=\mathrm{Holstein}, \mathrm{BRW}=\mathrm{Italian}$ Brown, $\mathrm{SIM}=\mathrm{Simmental}$, $\mathrm{MAR}=$ Marchigiana, PIE = Piedmontese, ED- $5 \%$ misAN = number of animals excluded with call rates $<95 \%, \mathrm{ED}-\mathrm{REPL}=$ number of animals excluded because they are replicates, ED- MEND $=$ number of animals excluded with Mendelian errors $>0.2 \%$.

\section{Comparison to previously reported data}

A number of studies have searched for selection sweeps in Holstein [2], Brown [20] and Simmental [21] cattle. Since different methods are expected to identify different signatures, comparison with previous results is limited to those using the same method and breed(s) as in our study. There is currently only one study that reported rEHH results in (German) Holstein-Friesian cattle [2]. The number of core haplotypes found in our study in the candidate regions was lower than that in Qanbari et al. [2] (Table 4). For Holsteins, the two most significant candidate regions in both studies agreed (casein gene cluster and somatostatin SST gene), although it is impossible to determine if the haplotype under selection is the same, since this information was not provided in [2]. However, other genes considered significant in Qanbari et al. [2] (with $p$-values ranging from 0.04 to $0.10)$ were not significant in our study. When using the same loose significance threshold as in Qanbari et al. [2] ( $p$-values $\leq 0.10)$, the casein gene cluster in this study was identified in Italian Brown $\left(-\log _{10} p\right.$-value $\left.=1.09\right)$ and the SST gene in Italian Simmental $\left(-\log _{10} p\right.$-value $\left.=1.26\right)$.

\section{Shared signatures between breeds}

Significant core haplotypes were aligned across breeds to identify those that were shared by dairy or beef breeds. Since breeds can be considered as independent sets of observations, shared signatures are more likely to represent real effects rather than false positives. A total of 123 significant core haplotypes (2.2\% of the genome), with an average length $216932 \mathrm{bp}$, were shared by at least two dairy breeds [See Additional file 2: Table S1]. For beef breeds, 142 core haplotypes (1.7\% of the genome) were shared by at least two breeds, with an average length of $190994 \mathrm{bp}$. Only 82 and 87 of the shared core haplotypes for dairy and beef breeds, respectively, contained genes. These were considered as positional candidate genes under positive selection and were further investigated.

\section{Gene set annotation and pathway analysis}

A total of 244 and 232 annotated genes fell within the regions under selection in dairy and beef breeds, respectively (Table 5 and [See Additional file 2: Table S1]). Among these, eight genes were shared by all three dairy breeds and 11 by all three beef breeds (see Figure 2 as an example).

All identified genes were submitted to pathway analyses. The most interesting genes for dairy breeds were breast cancer anti-estrogen resistance 3 (BARC3) and pituitary glutaminyl cyclase (QPCT), which are directly connected with the metabolism of the mammary gland $[22,23]$. Solute carrier family 2, member 5 (SLC2A5) facilitates glucose/fructose transport [24], and zeta-chain (TCR) associated protein kinase $70 \mathrm{kDa}(\mathrm{ZAP70})$ plays a critical role in T-cell signalling [25]. Calpain is another important complex that, together with calpain-3 (CAPN3), mediates epithelial-cell death during mammary gland involution [26]. Furthermore, RAS guanyl nucleotide-releasing protein (RASGRP1) activates the Erk/MAP kinase cascade,

Table 2 Comparison of rEHH signals in candidate regions in non-redundant and redundant dataset

\begin{tabular}{|c|c|c|c|c|c|c|c|}
\hline \multirow[t]{2}{*}{ Candidate region } & \multirow[t]{2}{*}{ BTA } & \multirow[t]{2}{*}{ Pos (bp) } & \multirow[t]{2}{*}{ Core haplotype range } & \multicolumn{2}{|c|}{ Redundant } & \multicolumn{2}{|c|}{ Non-redundant } \\
\hline & & & & CHFrq & rEHH $-\log (P)$ up $/$ down $^{1}$ & CHFrq & rEHH - $\log (P)$ up/down \\
\hline Polled locus & 1 & 1981154 & 1897418-1981154 & $\mathrm{H} 1: 0.79$ & $0.93 / 0.37$ & $\mathrm{H} 1: 0.78$ & $1.67^{*} / 1.74^{*}$ \\
\hline MC1R & 18 & 13657912 & 13317720-14007505 & $\mathrm{H1}: 0.69$ & $1.20 / 0.96$ & $\mathrm{H} 1: 0.69$ & $0.77 / 1.67^{*}$ \\
\hline KIT_BOVIN & 6 & 72821175 & $72504921-72821175$ & $\mathrm{H} 1: 0.54$ & $0.30 / 0.30$ & $\mathrm{H} 1: 0.54$ & $0.33 / 0.48$ \\
\hline \multirow[t]{2}{*}{ Casein cluster } & 6 & 88427760 & $88350095-88452829$ & $\mathrm{H} 1: 0.47$ & $0.94 / 1.39^{*}$ & $\mathrm{H} 1: 0.46$ & $1.48^{*} / 1.33^{*}$ \\
\hline & 6 & 88427760 & $88350095-88452829$ & $\mathrm{H} 2: 0.32$ & $0.02 / 0.03$ & $\mathrm{H} 2: 0.33$ & $0.02 / 0.03$ \\
\hline
\end{tabular}

$\mathrm{rEHH}$ in candidate regions in both uncorrected (redundant) and corrected (non-redundant) datasets for population structure; BTA = Bos taurus autosome; $\mathrm{CHFrq}=$ core haplotype frequency; ${ }^{1}$ up-stream (left) and down-stream (right) of the core haplotype; ${ }^{*}$ significant $\mathrm{rEHH}(\mathrm{p}<0.05$ ). 

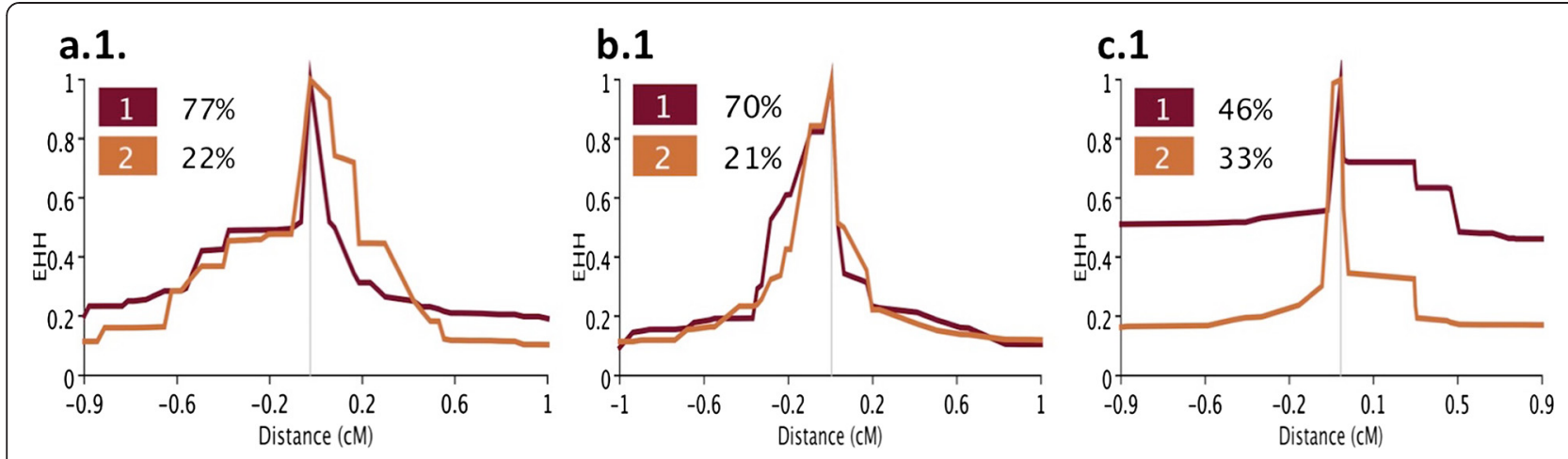

a.2.

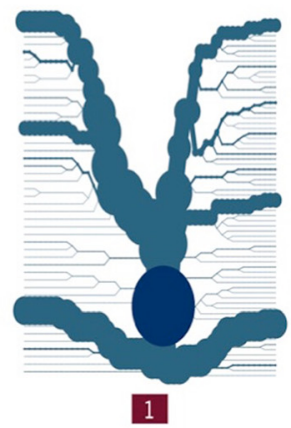

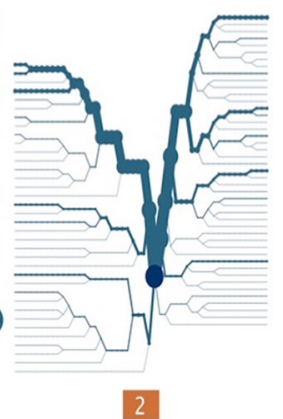

b. 2

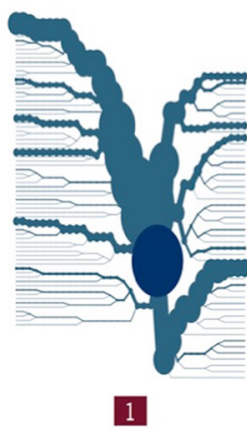

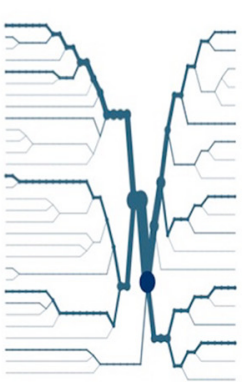

2

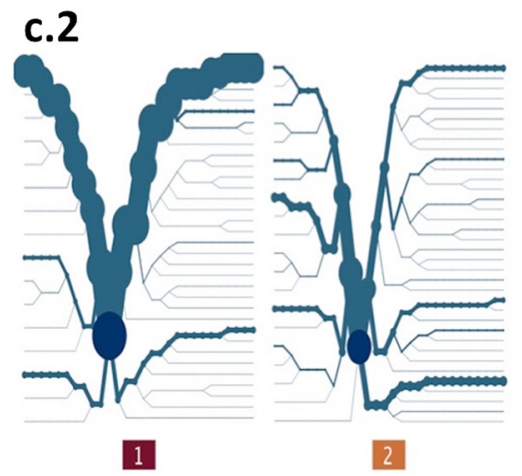

Figure 1 EHH decay over distance (1) and bifurcation plots (2) in the Italian Holstein non-redundant dataset. (a.1), (b.1) and (c.1) show the decay of haplotype homozygosity as a function of distance for the two most frequent core haplotypes. (a.2), (b.2) and (c.2) show haplotype bifurcation diagrams for the two most frequent core haplotypes at three control regions found to be significant rEHH in our study i.e. (a) polled locus, (b) MCIR gene and (c) casein gene cluster).

regulates the development of $\mathrm{T}$ - and B-cells, homeostasis and differentiation, and is involved in regulation of breast cancer cells [27-29].

Chondroitin sulfate proteoglycan 4 (CSPG4) and snurportin-1 (SNUPN) are the most interesting genes that were shared among all beef cattle breeds investigated. CSPG4 is related to meat tenderness, while SNUPN is an imprinted gene that has an important role in embryo development and is involved in human muscle atrophy [30].

A total of six and nine statistically significant canonical pathways $\left(\mathrm{FDR} \leq 0.05 ;-\log _{10}(\mathrm{FDR}) \geq 1.3\right.$ ) were identified using IPA for dairy and beef breeds, respectively (Figure 3 and [See Additional file 3: Table S2]). For the dairy breeds, the most significant canonical pathway was identified for purine metabolism $\left(-\log _{10}(F D R)=2.6\right)$, which supports the highly synthetic processes in the mammary epithelium [See Additional file 4: Figure S6]. In beef breeds, the signal for ephrin receptor $(-\log 10($ FDR $)=2.7)$ was the most significant canonical pathway [See Additional file 5: Figure S7]. Among other functions, ephrin receptor is known to promote muscle progenitor cell migration before mitotic activation [31]. All other canonical pathways are reported in Table S2 [See Additional file 3: Table S2].

\section{Discussion}

In this study, the genotypes of more than 4000 bulls from five Italian breeds were analysed for putative dairy and beef selection signatures. Strict data quality control was applied to reduce possible sources of bias from genotyping errors and population structure. In particular, the confounding effect of population structure was investigated by replicating part of the analyses without excluding a large number of close relatives and without balancing family members in the dataset. Assessment of the effect of population structure on $\mathrm{rEHH}$ results was restricted to four control regions that are known to be under selection in Italian Holstein, namely the casein gene cluster, the polled locus, and the MC1R and KIT genes. This breed was selected for two reasons: (i) according to our data it is a highly structured breed and (ii) it allowed comparing our results with a previous study [2]. Although analyses conducted on both redundant and non-redundant datasets identified rEHH signals in these regions, the non-redundant dataset produced five significant $\mathrm{rEHH}$ signals, compared to only one in the redundant dataset (Table 2). These results highlight the confounding effect of the presence of close relatives in the dataset and, consequently, the 
Table 3 Distribution of core haplotypes

\begin{tabular}{|c|c|c|c|c|c|c|c|c|c|c|}
\hline \multirow[t]{2}{*}{ BTA } & \multicolumn{2}{|c|}{ Italian Holstein } & \multicolumn{2}{|c|}{ Italian Brown } & \multicolumn{2}{|c|}{ Italian Simmental } & \multicolumn{2}{|c|}{ Marchigiana } & \multicolumn{2}{|c|}{ Piedmontese } \\
\hline & $\mathrm{Hap}^{2}$ & Sign.Hap ${ }^{3}$ & $\mathrm{Hap}^{2}$ & Sign.Hap ${ }^{3}$ & $\mathrm{Hap}^{2}$ & Sign.Hap ${ }^{3}$ & $\mathrm{Hap}^{2}$ & Sign.Hap ${ }^{3}$ & $\mathrm{Hap}^{2}$ & Sign.Hap ${ }^{3}$ \\
\hline 1 & 1238 & 66 & 1228 & 68 & 1062 & 49 & 988 & 51 & 907 & 46 \\
\hline 2 & 998 & 47 & 1040 & 52 & 830 & 44 & 822 & 42 & 735 & 33 \\
\hline 3 & 913 & 48 & 1039 & 62 & 780 & 46 & 721 & 34 & 702 & 34 \\
\hline 4 & 869 & 46 & 868 & 50 & 786 & 41 & 725 & 39 & 697 & 39 \\
\hline 5 & 696 & 32 & 680 & 29 & 619 & 30 & 543 & 27 & 488 & 22 \\
\hline 6 & 891 & 51 & 928 & 40 & 820 & 45 & 770 & 38 & 729 & 42 \\
\hline 7 & 761 & 37 & 807 & 46 & 631 & 39 & 624 & 33 & 661 & 27 \\
\hline 8 & 835 & 39 & 863 & 38 & 735 & 35 & 710 & 32 & 637 & 31 \\
\hline 9 & 738 & 37 & 727 & 41 & 649 & 37 & 532 & 22 & 575 & 30 \\
\hline 10 & 685 & 36 & 722 & 34 & 577 & 25 & 620 & 26 & 498 & 25 \\
\hline 11 & 816 & 39 & 758 & 34 & 686 & 30 & 637 & 33 & 586 & 25 \\
\hline 12 & 486 & 24 & 511 & 18 & 477 & 21 & 437 & 25 & 405 & 22 \\
\hline 13 & 675 & 32 & 602 & 19 & 477 & 18 & 539 & 35 & 424 & 19 \\
\hline 14 & 554 & 17 & 563 & 25 & 531 & 29 & 423 & 22 & 402 & 19 \\
\hline 15 & 571 & 31 & 581 & 29 & 468 & 23 & 446 & 13 & 399 & 19 \\
\hline 16 & 535 & 28 & 602 & 29 & 504 & 25 & 444 & 19 & 380 & 15 \\
\hline 17 & 524 & 27 & 585 & 29 & 540 & 31 & 429 & 22 & 389 & 16 \\
\hline 18 & 413 & 18 & 511 & 19 & 356 & 14 & 346 & 10 & 252 & 12 \\
\hline 19 & 424 & 22 & 436 & 23 & 349 & 16 & 310 & 18 & 295 & 15 \\
\hline 20 & 578 & 35 & 522 & 33 & 467 & 23 & 387 & 23 & 384 & 17 \\
\hline 21 & 458 & 23 & 478 & 30 & 356 & 18 & 315 & 21 & 312 & 13 \\
\hline 22 & 444 & 24 & 451 & 19 & 349 & 21 & 355 & 18 & 316 & 13 \\
\hline 23 & 325 & 13 & 315 & 16 & 198 & 10 & 186 & 9 & 182 & 8 \\
\hline 24 & 408 & 11 & 449 & 20 & 396 & 17 & 299 & 23 & 306 & 16 \\
\hline 25 & 273 & 12 & 271 & 11 & 205 & 10 & 246 & 12 & 214 & 6 \\
\hline 26 & 379 & 7 & 375 & 14 & 305 & 12 & 275 & 15 & 290 & 16 \\
\hline 27 & 284 & 12 & 281 & 15 & 235 & 11 & 268 & 12 & 190 & 10 \\
\hline 28 & 277 & 9 & 298 & 10 & 201 & 10 & 202 & 9 & 183 & 11 \\
\hline 29 & 315 & 15 & 310 & 13 & 248 & 10 & 215 & 9 & 209 & 12 \\
\hline тот & 17363 & 838 & 17801 & 866 & 14837 & 740 & 13814 & 692 & 12747 & 613 \\
\hline
\end{tabular}

Distribution of total and significant core haplotypes (up- and down-stream) per chromosome and breed; BTA = Bos taurus autosomes; ${ }^{2}$ number of detected core haplotypes with a frequency in the breed higher than $25 \%{ }^{3}$ number of significant core haplotypes at $p \leq 0.05$.

improved ability to detect a significant selection signature when correcting for population structure.

Due to pedigree links, population stratification rather than selection leads to an over-representation of haplotypes that are present in large families (e.g. sires that pass half of their genetic material to their sons). For this reason, for the full analyses, all sire-son pairs were removed after haplotype phasing (retaining only the sons), and half-sib families were restricted to a maximum of five randomly chosen individuals, to reduce family overrepresentation. This threshold was a compromise between limiting haplotype redundancy and retaining sufficient information to detect signals; reducing half-sib families to only one individual (which would have been the most rigorous choice), would have led to an excessive reduction of the dataset. The progeny-tested Italian bulls analysed in this study are highly related, especially those of the dairy type, and if the most stringent threshold had been applied, $82 \%$ of the Italian Holstein individuals would have been removed.

The three significant control regions (Table 2) in the non-redundant dataset showed a slightly different EHH decay over distance, as shown in the bifurcation plots of Figure 1. Here, EHH values are reported, since they are graphically easier to interpret. The two most frequent haplotypes for the polled locus showed a similar EHH 
Table 4 rEHH values in the candidate gene regions studied in [2]

\begin{tabular}{|c|c|c|c|c|c|c|c|c|c|c|c|}
\hline \multirow[b]{2}{*}{ Cand gene } & \multirow[b]{2}{*}{ BTA } & \multirow[b]{2}{*}{ Closest SNP (bp) } & \multicolumn{3}{|l|}{ Holstein } & \multicolumn{3}{|l|}{ Brown } & \multicolumn{3}{|l|}{ Simmental } \\
\hline & & & $\mathrm{CH}$ range & $\mathrm{CH}$ freq & rEHH-log(p) & $\mathrm{CH}$ range & $\mathrm{CH}$ freq & rEHH-log(p) & $\mathrm{CH}$ range & $\mathrm{CH}$ freq & rEHH-log(p) \\
\hline DGAT1 & 14 & 444963 & 236 653-443936 & $\mathrm{H} 1: 0.57$ & $-/ 0.11$ & $443936-763332$ & $\mathrm{H} 1: 0.42$ & $0.13 / 0.007$ & - & - & - \\
\hline \multirow[t]{3}{*}{ Casein cluster } & 6 & 88391612 & 88350 098-88 452835 & $\mathrm{H} 1: 0.46$ & $1.48^{*} / 1.33^{*}$ & $88326012-88452835$ & $\mathrm{H} 2: 0.68$ & $0.80 / 1.09$ & 88350 098-88 452835 & $\mathrm{H} 1: 0.44$ & $0.37 / 0.22$ \\
\hline & & & $88350098-88452835$ & $\mathrm{H} 2: 0.33$ & $0.18 / 0.30$ & - & - & - & - & - & - \\
\hline & & & - & - & - & - & - & - & $88350098-88452835$ & $\mathrm{H} 3: 0.34$ & $0.22 / 0.45$ \\
\hline GH & 19 & 49652377 & - & - & - & - & - & - & - & - & - \\
\hline GHR & 20 & 33908597 & - & - & - & - & - & - & - & - & - \\
\hline \multirow[t]{2}{*}{ SST } & 1 & 81376956 & $81283585-81376961$ & $\mathrm{H} 1: 0.36$ & $2.00^{* *} / 1.89^{* *}$ & - & - & - & $81318451-81376961$ & $\mathrm{H} 1: 0.42$ & $1.26 / 0.53$ \\
\hline & & & $81283585-81376961$ & $\mathrm{H} 2: 0.29$ & $0.063 / 0.084$ & - & - & - & $81318451-81376961$ & $\mathrm{H} 2: 0.42$ & $0.06 / 0.27$ \\
\hline IGF-1 & 5 & 71169823 & - & - & - & - & - & - & - & - & - \\
\hline \multirow[t]{2}{*}{ ABCG2 } & 6 & 37374911 & - & - & - & 37317 020-38 256889 & $\mathrm{H} 1: 0.44$ & $0.31 / 0.27$ & - & - & - \\
\hline & & & - & - & - & 37317 020-38 256889 & $\mathrm{H} 1: 0.40$ & $0.29 / 0.31$ & - & - & - \\
\hline Leptin & 4 & 95715500 & - & - & - & - & - & - & - & - & - \\
\hline \multirow[t]{2}{*}{ LPR } & 3 & 85569203 & 85497 108-85 594551 & $\mathrm{H} 1: 0.47$ & $0.91 / 0.72$ & 85497 108-85 794693 & $\mathrm{H} 1: 0.68$ & $0.80 / 0.63$ & 85497 108-85 794693 & $\mathrm{H} 1: 0.63$ & $0.02 / 0.05$ \\
\hline & & & 85497 108-85 594551 & $\mathrm{H} 2: 0.41$ & $0.27 / 0.25$ & - & - & - & - & - & - \\
\hline PIT-1 & 1 & 35756434 & - & - & - & - & - & - & - & - & - \\
\hline
\end{tabular}


Table 5 Statistics on common significant core haplotypes in dairy and beef breeds

\begin{tabular}{|c|c|c|c|c|c|c|c|c|}
\hline \multirow[b]{2}{*}{ BTA $^{1}$} & \multicolumn{2}{|c|}{ Dairy breeds } & \multirow[b]{2}{*}{ Sum CH size ${ }^{4}$} & \multirow[b]{2}{*}{ Avg. CH size ${ }^{5}$} & \multicolumn{2}{|c|}{ Beef breeds } & \multirow[b]{2}{*}{ Sum $\mathrm{CH}$ size ${ }^{4}$} & \multirow[b]{2}{*}{ Avg. $\mathrm{CH}$ size } \\
\hline & Sign. $\mathrm{CH}^{2}$ & $\mathrm{Nb}_{\text {genes }}{ }^{3}$ & & & Sign. $\mathrm{CH}^{2}$ & $\mathrm{Nb}_{\text {genes }}{ }^{3}$ & & \\
\hline 1 & 7 & 11 & 1521608 & 138328 & 7 & 13 & 2263213 & 174093 \\
\hline 2 & 5 & 9 & 2216685 & 246298 & 8 & 11 & 2658549 & 241686 \\
\hline 3 & 2 & 5 & 1619336 & 323867 & 7 & 21 & 3755847 & 178850 \\
\hline 4 & 7 & 21 & 5956755 & 283655 & 6 & 11 & 1761288 & 160117 \\
\hline 5 & 4 & 17 & 4506119 & 265066 & 5 & 11 & 1251127 & 113739 \\
\hline 6 & 2 & 4 & 1467738 & 366934 & 5 & 12 & 5149692 & 429141 \\
\hline 7 & 6 & 30 & 4842969 & 161432 & 3 & 28 & 11889191 & 424614 \\
\hline 8 & 0 & 0 & 0 & 0 & 4 & 12 & 3512215 & 292685 \\
\hline 9 & 4 & 7 & 1554863 & 222123 & 2 & 6 & 1106768 & 184461 \\
\hline 10 & 4 & 17 & 8839762 & 519986 & 2 & 3 & 573138 & 191046 \\
\hline 11 & 6 & 8 & 1841802 & 230225 & 1 & 1 & 100439 & 100439 \\
\hline 12 & 2 & 8 & 4244133 & 530517 & 1 & 1 & 536461 & 536461 \\
\hline 13 & 3 & 8 & 1933026 & 241628 & 2 & 8 & 985376 & 123172 \\
\hline 14 & 0 & 0 & 0 & 0 & 3 & 9 & 692397 & 76933 \\
\hline 15 & 5 & 9 & 1415405 & 157267 & 2 & 2 & 189094 & 94547 \\
\hline 16 & 3 & 6 & 1302506 & 217084 & 4 & 6 & 905719 & 150953 \\
\hline 17 & 3 & 4 & 471046 & 117762 & 2 & 9 & 1551010 & 172334 \\
\hline 18 & 0 & 0 & 0 & 0 & 3 & 23 & 2961718 & 128770 \\
\hline 19 & 3 & 23 & 5744143 & 249745 & 2 & 2 & 116938 & 58469 \\
\hline 20 & 1 & 2 & 148886 & 74443 & 2 & 4 & 627971 & 156993 \\
\hline 21 & 3 & 7 & 1930206 & 275744 & 1 & 5 & 1150760 & 230152 \\
\hline 22 & 3 & 5 & 620674 & 124135 & 3 & 10 & 2411751 & 241175 \\
\hline 23 & 0 & 0 & 0 & 0 & 1 & 1 & 68421 & 68421 \\
\hline 24 & 2 & 18 & 9166004 & 509222 & 2 & 4 & 803770 & 200942 \\
\hline 25 & 2 & 11 & 2016602 & 183327 & 1 & 3 & 329199 & 109733 \\
\hline 26 & 2 & 4 & 466134 & 116534 & 4 & 7 & 776080 & 110869 \\
\hline 27 & 1 & 1 & 631792 & 631792 & 2 & 3 & 1004717 & 334906 \\
\hline 28 & 0 & 0 & 0 & 0 & 1 & 1 & 93464 & 93464 \\
\hline 29 & 2 & 9 & 935209 & 103912 & 1 & 5 & 798300 & 159660 \\
\hline тот & 82 & 244 & 65393403 & 216932 & 87 & 232 & 50024613 & 190994 \\
\hline
\end{tabular}

${ }^{1}$ Bos taurus autosomes; ${ }^{2}$ number of significant core haplotypes $(P<0.05) ;{ }^{3}$ number of genes identified in the significant regions; ${ }^{4}$ sum of significant core haplotypes size, in bp; ${ }^{5}$ average size of significant core haplotypes, in bp.

pattern: high values (i.e. $\sim 1$ ) close to the core haplotype and a rapid decay to 0.2 at $\sim 1 \mathrm{cM}$ down- and up-stream (Figure 1.a). The second haplotype, however, was excluded from this analysis, since its frequency was lower than the threshold that was set $(<25 \%)$. Interestingly, the cumulative frequency of these two haplotypes in the whole Italian Holstein population was 99\%, e.g. nearly all individuals carried these two (core) haplotypes. A similar pattern was observed for the two most frequent haplotypes in the $M C 1 R$ gene (Figure $1 \mathrm{~b}$ ). In contrast to the polled locus, high EHH values (e.g. > 0.5) were maintained at distances of more than $200 \mathrm{~kb}$ up- and downstream from the core haplotype for the $M C 1 R$ gene, which potentially indicates more recent and strong selection. A more conserved haplotype was particularly evident for the casein gene cluster (Figure 1c), with $\mathrm{EHH}$ values greater than 0.6 at distances of more than $\sim 1 \mathrm{Mb}$ up- and down-stream from the core haplotype. Interestingly, similar values (both in terms of haplotype frequency and $\mathrm{EHH}$ ) were reported in [2].

We also compared results of all candidate regions investigated in [2], our results only partially overlapped with those reported by Qanbari et al. [2]. Common signals were found at the casein gene cluster (see above) and the SST gene, while Qanbari et al. [2] found significant signals also in other regions. These inconsistencies 

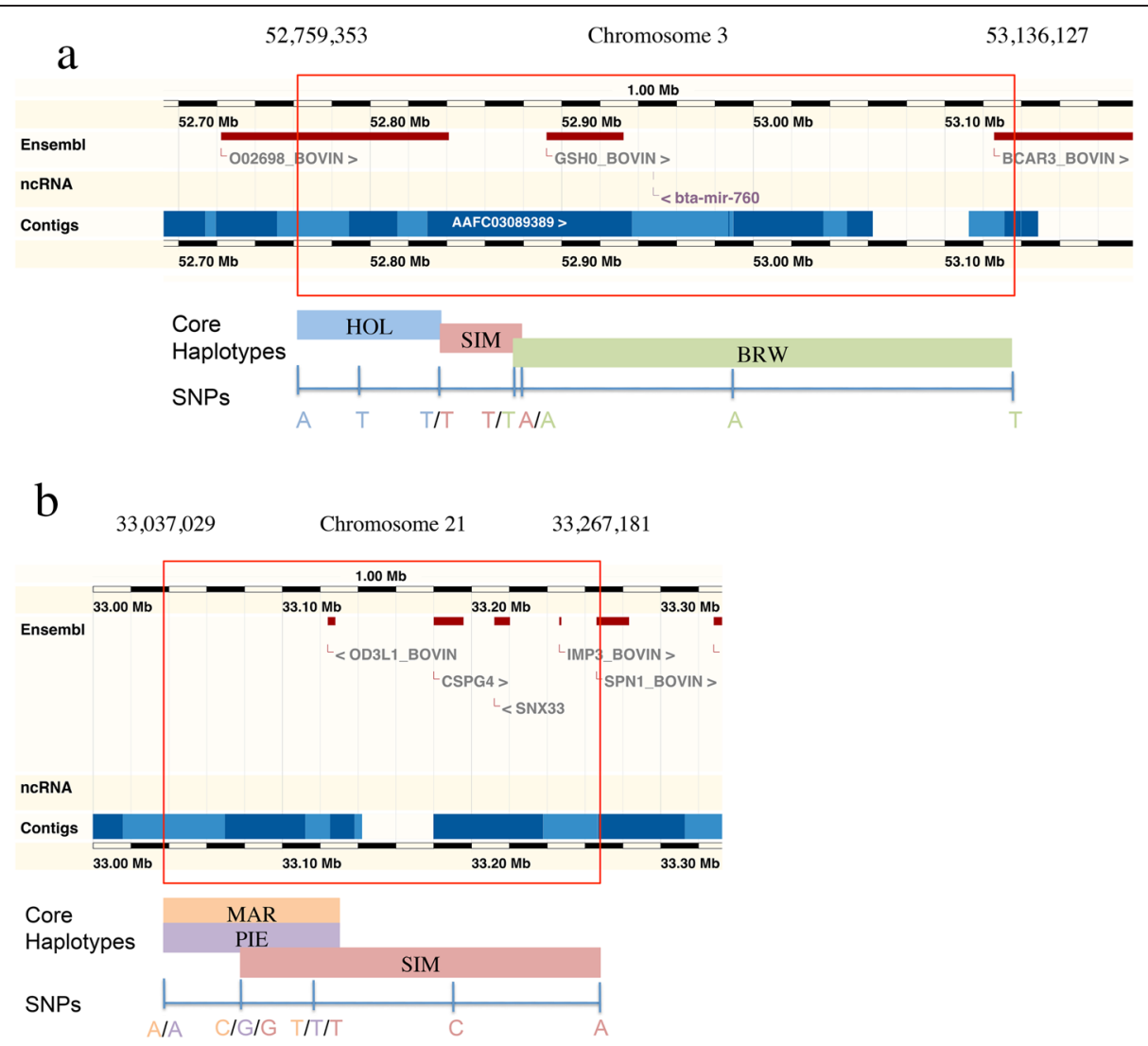

Figure 2 Genomic location of the selection signatures shared among the studied breeds. (a) Genes in Ensembl tracks are displayed as red boxes; core haplotypes and SNPs are coloured in orange (Marchigiana; MAR), in purple (Piedmontese; PIE) and pink (Simmental; SIM). (b) Genes in Ensembl tracks are displayed as red boxes; core haplotypes and SNPs are coloured in blue (Holstein; HOL), in green (Italian Brown; BRW) and pink (Simmental; SIM).

may be due to the presence of different sires in the analyses, different dataset sizes, or to the close relative reduction procedure that we adopted to decrease the effect of population structure and consequent bias. However, poor agreement across studies is similarly observed in human studies and is often due to: (i) use of different within- and between-populations statistics that potentially identify selection signatures with different characteristics (ancient/recent, segregating/fixed, under directional/balancing selection), (ii) high rate of false positive/negative results, and (iii) different ways of accounting for population structure and background selection [32]. In a recent study, Mancini et al. [33] estimated the fixation index (Fst) in the same populations here investigated and identified signals that do not overlap with those reported here. Although at least a partial overlap was expected, this could be explained by the intrinsic differences between Fst and rEHH methods. By comparing two populations (or groups of populations), Fst is much more efficient in capturing large allele frequency differences between breeds and thus identifies "outlier" SNPs that are fixed or close to fixation for opposite alleles. This means that the identified signals are usually markers that have been differentially selected for a relatively large number of generations (e.g. "old" selection). Conversely, the rEHH identifies long haplotypes that segregate at high frequency in the population, and thus are, by definition, recent.

The number of total and significant core haplotypes identified by the Sweep software was highest for the Italian Brown and lowest for the Piedmontese breed. Since rEHH methods rely heavily on population LD, the average LD at different genomic distances was estimated for each breed (Figure 4). Although values of LD based on sequence data decay at shorter distances than the values presented here [34], this analysis highlighted a general positive correlation between the level of LD over distance and the number of total and significant core haplotypes found. However, considering that $\mathrm{rEHH}$ is a relative measure, the larger number of significant core haplotypes identified for dairy breeds was likely due to the higher selective pressure (and thus a higher local LD at specific loci) in dairy compared to beef breeds. 

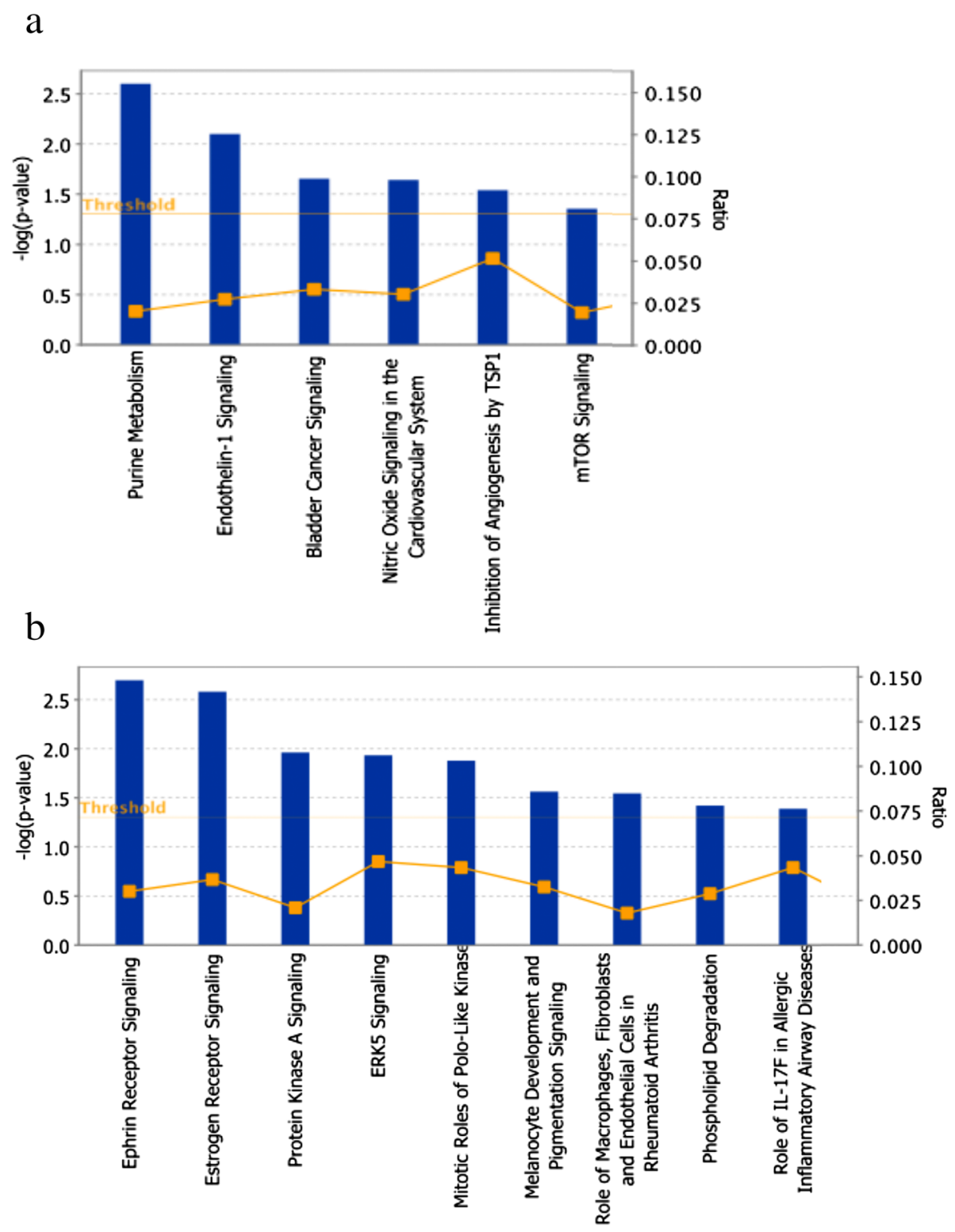

Figure 3 Bar plot of statistically significant canonical pathways. $P$-values were corrected for multiple-testing using the Benjamini-Hochberg method and are presented in the graph as - $\log (p$-value). The bar represents the percentage of genes in a given pathway that meet the cut-off criteria within the total number of molecules that belong to the function. (a) Bar plot of statistically significant canonical pathways in dairy cattle breeds. (b) Bar plot of statistically significant canonical pathways in beef cattle breeds.

Significance tests used to detect selection signatures should measure the probability of a statistic being an outlier value compared to its expected distribution under a neutral model. However, no reliable neutral model has so far been developed for cattle because of the complexity of the demographic history of this species [13]. As a consequence, empirical rather than model-based significance tests are generally used to detect selection signatures. Accordingly, we considered outlier values as those falling in the $5 \%$ plus variant tail of the rEHH distribution. We kept the within-breed significance threshold loose without correcting it for multiple-testing, but considered only signals shared by two or more independent breeds of the same production type.

The parallel comparison of results from independent analyses of different breeds allowed us to reach a double objective: (i) identification of putative regions under (recent) selection in breeds with different production purposes, which was the main objective of this study, and (ii) reduction of the rate of false positives, since multibreed analyses served as internal controls. Since the 


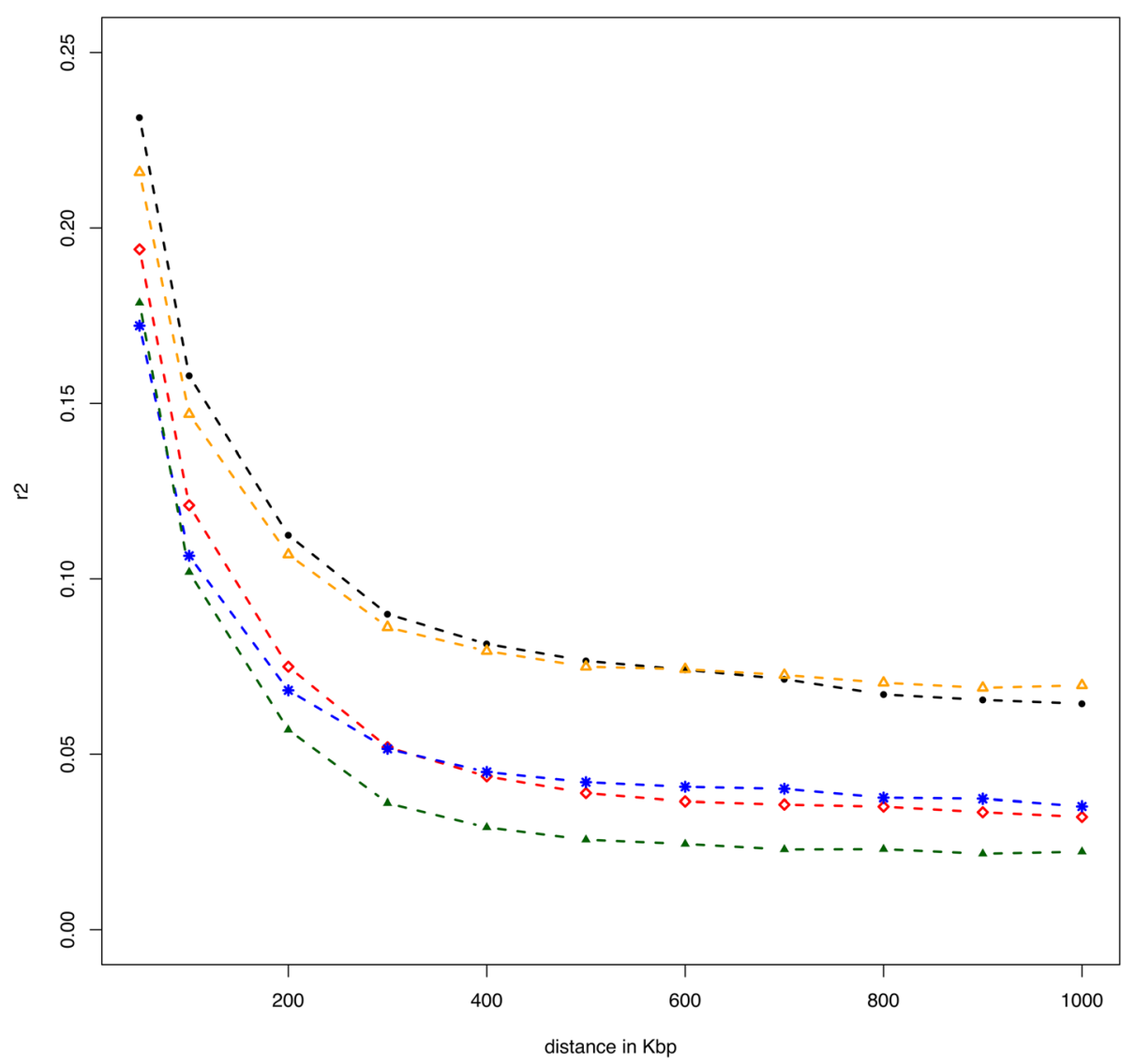

Figure 4 Multi-breed average linkage disequilibrium against physical distance (in kb). Marchigiana (blue stars), Piedmontese (green filled triangles) and Italian Simmental (red diamonds) breeds show a lower persistence of LD over distance than Italian Holstein (black filled circles) and Italian Brown (orange triangles) breeds.

rEHH method does not consider phenotypic information, a significant signal might arise because: (i) the core haplotype is actually under selective pressure or (ii) the result is a false positive, i.e. caused by chance, population structure or any other driving force. However, even considering an unrealistic scenario with no false positives, a proportion of all signals will actually be selection signatures due to selection pressure on traits other than those specific to dairy or beef traits. This is because only a few dairy and beef breeds were analysed and beef breeds share a number of traits that are not directly related to dairy or beef production, such as coat colour, polled/horned, etc. Even considering this limitation, to our knowledge, this is the first multi-breed study in dairy and beef cattle that applies such a strategy to reduce the rate of false positives, at the cost of a possible loss of information due to higher false negative rates. Significant signals shared by dairy and beef breeds were used for downstream gene annotation and pathway analyses on positional candidate genes to investigate the biological processes behind the genomic signals. Only the most significant pathways for dairy and beef breeds will be discussed in detail in the following.

\section{Dairy breeds}

Putative signals of selection were found in regions that contain the BARC3 and QPCT genes, and these were shared among all three dairy breeds. To date, neither of these genes have been studied in cattle. However, human studies have shown that these genes are linked to mammary gland metobolism and calcium regulation. BARC3 is involved in integrin-mediated cell adhesion and signaling, which is required for mammary gland development and function [23]. QPCT is associated with low radial body mineral density (BMD) in adult women [22]. Another interesting candidate gene is $S L C 2 A 5$, which acts as a fructose transporter in the intestine and has a significant role in energy balance of dairy cows [35]. The detection of this gene in a dairy cattle specific candidate region is surprising, since, in theory, there should be little need for transporting glucose and/or fructose in the ruminant intestinal tract because simple carbohydrates are degraded into volatile fatty acids (VFA) in the rumen [36]. However, it is known that large amounts of starch bypass the rumen in cows fed on diets that are rich in cereal grains [24]. This bypassed starch needs to be 
digested in the small intestine and then absorbed, to avoid high levels of glucose in the large intestine.

We detected candidate regions that contained the calpain complex and calpain-3 (CAPN3) genes in the dairy breeds, as reported by Utsunomiya et al. [7]. Although calpain is known to be involved in postmortem meat tenderization, it is also related to dairy metabolism, since muscle breakdown promoted by calpain provides an energy source for milk production especially at the beginning of lactation [37]. In addition, as reported by Wilde et al. [26], the calpain-calpastatin system is related to the programmed cell death of alveolar secretory epithelial cells during lactation. The Zap70 gene encodes a cytoplasmic protein tyrosine kinase that is related to the immune system and plays a central role in T-cell responses, as a component of the T-cell receptor [38]. Bonnefont et al. [25] reported that the Zap70 gene was up-regulated in somatic cells present in the milk of sheep infected by Staphylococcus aureus and Staphylococcus epidermidis, which suggests an association with mastitis resistance.

Purine metabolism was the most significant canonical pathway in dairy breeds [See Additional file 4: Figure S6]. In a gene expression analysis on human breast milk fat globules, Maningat et al. [39] identified purine metabolism as the most significant pathway. Synthesis and breakdown of purine is essential in the tissue metabolism of many organisms, and in particular in that of the mammary gland during lactation.

Another interesting canonical pathway was endothelin signalling (Figure 3). Endothelin functions as a vasoconstrictor and is secreted by endothelial cells [40]. Acosta et al. [41] reported that in cattle, endothelins are involved in the follicular production of prostaglandins and the regulation of steroidogenesis in the mature follicle. In a recent study, Puglisi et al. [42] confirmed that endothelins, in particular EDNRA (a potential biomarker for fertility in cow) and endothelin convertin enzyme 1, are involved in a reproductive disorder in cows.

\section{Beef breeds}

A suggestive signature of selection in all three beef breeds was found in the region of the CSPG4 gene, which belongs to the chondroitin sulfate proteoglycan (CSPG) gene family. CSPG are proteoglycans that consist of a protein core and a chondroitin sulfate side chain. They are known to be structural components of a variety of tissues, including muscle, and to play key roles in neural development and glial scar formation. They are involved in cellular processes, such as cell adhesion, cell growth, receptor binding, cell migration, and interactions with other extracellular matrix constituents.

Many studies have reported the role of proteoglycans in the determination of meat texture of several bovine muscles [43]. Dubost et al. [44] highlighted a direct role of proteoglycans in cooked meat juiciness. Another putative signal of selection was found on the RB1-inducible coiled-coil 1 (RB1CC1) gene. This gene plays a crucial role in muscular differentiation and its activation is essential for myogenic differentiation [45]. The monoacylglycerol acyltransferase (MGAT3) gene catalyses the synthesis of diacylglycerol (DAG) using 2-monoacylglycerol and fatty acyl coenzyme A. This enzymatic reaction is fundamental for the absorption of dietary fat in the small intestine. In a study on five Chinese cattle breeds, Sun et al. [46] reported that the MGAT3 gene is associated with growth traits. The cold inducible RNA binding protein (CIRPB) gene may be part of a compensatory mechanism in muscles that undergo atrophy. It preserves muscle tissue mass during cold-shock responses, aging and disease [47]. SNUPN is an imprinted gene that is expressed monoallelically, depending on its parental origin. SNUPN plays important roles in embryo survival and postnatal growth regulation $[48,49]$. Ephrin receptor signalling was the top canonical pathway identified by IPA and has interesting biological roles for meat production [See Additional file 5: Figure S7]. Indeed, this pathway is important for muscle tissue growth and regeneration by participating in the correct positioning and formation of the neural muscular junction [31].

\section{Conclusions}

In this study, we analysed candidate selection signatures at the genome-wide level in five Italian cattle breeds. Then, we used a multi-breed approach to identify the genomic regions shared among cattle breeds selected for dairy or beef production. This approach increased the potential of pin-pointing regions of the genome that play important roles in economically relevant traits. Moreover, gene annotation and pathway analyses were used to describe the gene functions in the regions potentially under recent positive selection.

Specifically, dairy cattle genes that are likely to be under directional selection are related to feeding adaptation (increasing levels of starch in the diet), mammary gland metabolism and resistance to mastitis, while putative regions under selection in beef cattle are related to animal growth, meat texture and juiciness. Considering that annotation for the bovine genome is not as accurate as for the human genome, the biological interpretation of selection signatures can be derived based only on genes that are located near candidate regions. Moreover, novel information in humans suggests that many selected variations are not located within genes and coding regions, but in regulatory sites that have been identified within the ENCODE project [32]. These may control the expression of entire genomic regions or genes located at a relevant distance 
from the selected site, making biological interpretation more complex.

Future studies using denser SNP chips or wholegenome sequencing that provide information not subjected to ascertainment bias [34], may increase the resolution of our analysis and, together with increasing knowledge on the control of gene expression, should validate our results.

\section{Additional files}

\section{Additional file 1: Figures S1, S2, S3, S4 and S5. Genome-wide map} of P-values for core haplotypes for Italian Holstein, Italian Brown, Italian Simmental, Marchigiana and Piedmontese breeds, respectively. The file is a .zip compressed document including five .tiff images i.e. Figures S1, S2, S3, S4 and S5 that show the genome-wide map of P-values for core haplotypes with a frequency higher than 0.25 for Italian Holstein (HOL), Italian Brown (BRW), Italian Simmental (SIM), Marchigiana (MAR) and Piedmontese (PIE) breeds, respectively. Dashed lines represent the cut-off level of 0.01

Additional file 2: Table S1. Significant core haplotypes and genes shared between dairy and beef cattle breeds. Significant core haplotypes ( $p$-value $\leq 0.05$; haplotype frequency $\geq 0.25$ ) shared among dairy and beef cattle breeds and the relative genes that intersect with the core haplotypes. The file is a .xls document that includes four sheets. The first two sheets show the significant core haplotypes for dairy and beef cattle breeds and the other two sheets show genes intersecting with the significant core haplotypes for dairy and beef cattle breeds.

Additional file 3: Table S2. Ranking of canonical pathways in dairy or beef cattle breeds. Ranking of canonical pathways in dairy or beef cattle breeds with the list of corresponding gene symbols, ratio and $-\log 10$ of the $p$-values for each canonical pathway.

Additional file 4: Figure $\mathbf{S 6}$. Genes detected under recent positive selection in dairy cattle and involved in the purine metabolism canonical pathway. Description: In Figure S6, nodes in red correspond to genes identified in core haplotypes that overlap in all three breeds of each production type, whereas those in green depict overlapping core haplotypes in at least two of those breeds.

Additional file 5: Figure S7. Genes detected under recent positive selection in beef cattle and involved in the ephrin metabolism canonical pathway. In Figure S7, nodes in red correspond to genes identified in core haplotypes that overlap in all three breeds of each production type, whereas those in green depict overlapping core haplotypes in at least two of those breeds.

\section{Competing interests}

The authors declare that they have no competing interests.

\section{Authors' contributions}

PAM, AV, AS, LB and ELN and RN contributed to the design of the study. $L B$ ELN, MM, GM and FB generated the data and performed data analyses. $L B$ and ELN drafted the manuscript. PAM, LB, ELN, MM and RN interpreted the results and contributed to the editing of the manuscript. All authors read and approved the final manuscript.

\section{Acknowledgements}

ANAFI (Italian Association of Holstein Friesian Breeders), ANAPRI (Italian Association Simmental Breeders), ANABORAPI (National Association of Piedmontese Breeders), ANABIC (Association of Italian Beef Cattle Breeders) and ANARB (Italian Brown Cattle Breeders Association) are acknowledged for providing the biological samples and the necessary information for this work. The authors are also grateful to the "SelMol" project (MiPAAF, Ministero delle Politiche Agricole, Alimentari e Fortestali) and the "ProZoo" project (Regione Lombardia-DG Agricoltura, Fondazione Cariplo, Fondazione Banca Popolare di Lodi) for funding. The funders had no role in the design of the study, data collection and analysis, decision to publish, or preparation of the manuscript.

\section{Author details}

'Istituto di Zootecnica, UCSC, via Emilia Parmense 84, Piacenza 29122, Italy. ${ }^{2}$ Fondazione Parco Tecnologico Padano, Via Einstein, Loc. Cascina Codazza, Lodi 26900, Italy. ${ }^{3}$ Associazione Italiana Allevatori (AIA), Via Tomassetti 9, Rome 00161, Italy. ${ }^{4}$ Center for Computational Chemistry and Cosmology, Scuola Normale Superiore, Via Consoli del Mare 2, Pisa 56126, Italy. ${ }^{5}$ Istituto di biologia e biotecnologia Agraria (IBBA-CNR), Consiglio Nazionale delle Ricerche, Via Einstein, Cascina Codazza, Lodi 26900, Italy. ${ }^{6}$ Dipartimento per I'Innovazione nei Sistemi Biologici, Agroalimentari e Forestali (DIBAF), via de Lellis, Viterbo 01100, Italy.

Received: 1 August 2014 Accepted: 19 March 2015

Published online: 02 April 2015

\section{References}

1. Hayes BJ, Lien S, Nilsen H, Olsen HG, Berg P, Maceachern S, et al. The origin of selection signatures on bovine chromosome 6. Anim Genet. 2008;39:105-11.

2. Qanbari S, Pimentel ECG, Tetens J, Thaller G, Lichtner P, Sharifi AR, et al. A genome-wide scan for signatures of recent selection in Holstein cattle. Anim Genet. 2010;41:377-89.

3. Shahzad K, Loor JJ. Application of top-down and bottom-up systems approaches in ruminant physiology and metabolism. Curr Genomics. 2012;13:379-94.

4. Nielsen R, Hellmann I, Hubisz M, Bustamante C, Clark AG. Recent and ongoing selection in the human genome. Nat Rev Genet. 2007;8:857-68.

5. Lenstra JA, Groeneveld LF, Eding H, Kantanen J, Williams JL, Taberlet $\mathrm{P}$, et al. Molecular tools and analytical approaches for the characterization of farm animal genetic diversity. Anim Genet. 2012;43:483-502.

6. Pérez O’Brien AM, Utsunomiya YT, Mészáros G, Bickhart DM, Liu GE, Tassell $C P V$, et al. Assessing signatures of selection through variation in linkage disequilibrium between taurine and indicine cattle. Genet Sel Evol. 2014:46:19.

7. Utsunomiya YT, Perez O'Brien AM, Sonstegard TS, Van Tassell CP, do Carmo AS, Mészáros $G$, et al. Detecting loci under recent positive selection in dairy and beef cattle by combining different genome-wide scan methods. PLoS ONE. 2013;8:e64280.

8. Sabeti PC, Reich DE, Higgins JM, Levine HZ, Richter DJ, Schaffner SF, et al. Detecting recent positive selection in the human genome from haplotype structure. Nature. 2002;419:832-7.

9. Pan D, Zhang S, Jiang J, Jiang L, Zhang Q, Liu J. Genome-wide detection of selective signature in Chinese Holstein. PLoS ONE. 2013;8:e60440.

10. Zhang C, Bailey DK, Awad T, Liu G, Xing G, Cao M, et al. A whole genome long-range haplotype (WGLRH) test for detecting imprints of positive selection in human populations. Bioinformatics. 2006:22:2122-8.

11. Kimura M. The neutral theory of molecular evolution. Cambridge: Cambridge University Press; 1984

12. Voight BF, Kudaravalli S, Wen X, Pritchard JK. A map of recent positive selection in the human genome. PLoS Biol. 2006:4:e72.

13. Kemper KE, Saxton SJ, Bolormaa S, Hayes BJ, Goddard ME. Selection for complex traits leaves little or no classic signatures of selection. BMC Genomics. 2014;15:246.

14. Ma $Y L$, Zhang $Q$, Ding XD. Detecting selection signatures on $X$ chromosome in pig through high density SNPs]. [Article in Chinese. Yi Chuan. 2012;34:1251-60.

15. Taberlet P, Coissac E, Pansu J, Pompanon F. Conservation genetics of cattle, sheep, and goats. C R Biol. 2011;334:247-54.

16. Ajmone-Marsan P, Garcia JF, Lenstra JA. On the origin of cattle: How Aurochs became cattle and colonized the world. Evol Anthropol. 2010;19:148-57.

17. Hayes BJ, Chamberlain AJ, Maceachern S, Savin K, McPartlan H, MacLeod I, et al. A genome map of divergent artificial selection between Bos taurus dairy cattle and Bos taurus beef cattle. Anim Genet. 2009:40:176-84.

18. Scheet P, Stephens M. A fast and flexible statistical model for large-scale population genotype data: applications to inferring missing genotypes and haplotypic phase. Am J Hum Genet. 2006;78:629-44.

19. Purcell $\mathrm{S}$, Neale B, Todd-Brown K, Thomas L, Ferreira MAR, Bender D, et al. PLINK: a tool set for whole-genome association and population-based linkage analyses. Am J Hum Genet. 2007:81:559-75.

20. Qanbari S, Gianola D, Hayes B, Schenkel F, Miller S, Moore S, et al. Application of site and haplotype-frequency based approaches for detecting selection signatures in cattle. BMC Genomics. 2011;12:318. 
21. Fan H, Wu Y, Qi X, Zhang J, Li J, Gao X, et al. Genome-wide detection of selective signatures in Simmental cattle. J Appl Genet. 2014;55:343-51.

22. Ezura Y, Kajita M, Ishida R, Yoshida S, Yoshida H, Suzuki T, et al. Association of multiple nucleotide variations in the pituitary glutaminyl cyclase gene (QPCT) with low radial BMD in adult women. J Bone Miner Res. 2004;19:1296-301.

23. Sun G, Cheng SYS, Chen M, Lim CJ, Pallen CJ. Protein tyrosine phosphatase alpha phosphotyrosyl-789 binds BCAR3 to position Cas for activation at integrin-mediated focal adhesions. Mol Cell Biol. 2012;32:3776-89.

24. Zhao FQ, Okine EK, Cheeseman Cl, Shirazi-Beechey SP, Kennelly JJ. Glucose transporter gene expression in lactating bovine gastrointestinal tract. J Anim Sci. 1998;76:2921-9.

25. Bonnefont CMD, Toufeer M, Caubet C, Foulon E, Tasca C, Aurel MR, et al. Transcriptomic analysis of milk somatic cells in mastitis resistant and susceptible sheep upon challenge with Staphylococcus epidermidis and Staphylococcus aureus. BMC Genomics. 2011;12:208.

26. Wilde CJ, Addey CV, Li P, Fernig DG. Programmed cell death in bovine mammary tissue during lactation and involution. Exp Physiol. 1997;82:943-53.

27. Madani S, Hichami A, Charkaoui-Malki M, Khan NA. Diacylglycerols containing omega 3 and omega 6 fatty acids bind to RasGRP and modulate MAP kinase activation. J Biol Chem. 2004;279:1176-83.

28. Yasuda S, Stevens RL, Terada T, Takeda M, Hashimoto T, Fukae J, et al. Defective expression of Ras guanyl nucleotide-releasing protein 1 in a subset of patients with systemic lupus erythematosus. J Immunol. 2007;179:4890-900.

29. Wickramasinghe NS, Manavalan T, Dougherty SM, Riggs KA, Li Y, Klinge CM. Estradiol downregulates miR-21 expression and increases miR-21 target gene expression in MCF-7 breast cancer cells. Nucleic Acids Res. 2009;37:2584-95

30. Narayanan U, Ospina JK, Frey MR, Hebert MD, Matera AG. SMN, the spinal muscular atrophy protein, forms a pre-import snRNP complex with snurportin1 and importin beta. Hum Mol Genet. 2002;11:1785-95.

31. Li J, Johnson SE. Ephrin-A5 promotes bovine muscle progenitor cell migration before mitotic activation. J Anim Sci. 2013;91:1086-93.

32. Enard D, Messer PW, Petrov DA. Genome-wide signals of positive selection in human evolution. Genome Res. 2014;24:885-95.

33. Mancini G, Gargani M, Chillemi G, Nicolazzi EL, Marsan PA, Valentini A, et al. Signatures of selection in five Italian cattle breeds detected by a 54 K SNP panel. Mol Biol Rep. 2014;41:957-65.

34. Qanbari S, Pausch H, Jansen S, Somel M, Strom TM, Fries R, et al. Classic selective sweeps revealed by massive sequencing in cattle. PLoS Genet. 2014;10:e1004148.

35. Burant CF, Takeda J, Brot-Laroche E, Bell Gl, Davidson NO. Fructose transporter in human spermatozoa and small intestine is GLUT5. J Biol Chem. 1992;267:14523-6.

36. Iqbal S, Zebeli Q, Mazzolari A, Bertoni G, Dunn SM, Yang WZ, et al. Feeding barley grain steeped in lactic acid modulates rumen fermentation patterns and increases milk fat content in dairy cows. J Dairy Sci. 2009;92:6023-32.

37. Kuhla B, Nürnberg G, Albrecht D, Görs S, Hammon HM, Metges CC. Involvement of skeletal muscle protein, glycogen, and fat metabolism in the adaptation on early lactation of dairy cows. J Proteome Res. 2011;10:4252-62.

38. Wang H, Kadlecek TA, Au-Yeung BB, Goodfellow HES, Hsu LY, Freedman TS, et al. ZAP-70: an essential kinase in T-cell signaling. Cold Spring Harb Perspect Biol. 2010;2:a002279.

39. Maningat PD, Sen P, Rijnkels $M$, Sunehag AL, Hadsell DL, Bray M, et al. Gene expression in the human mammary epithelium during lactation: the milk fat globule transcriptome. Physiol Genomics. 2008;37:12-22.

40. Nussdorfer GG, Rossi GP, Malendowicz LK, Mazzocchi G. Autocrine-paracrine endothelin system in the physiology and pathology of steroid-secreting tissues. Pharmacol Rev. 1999;51:403-38.

41. Acosta TJ, Berisha B, Ozawa T, Sato K, Schams D, Miyamoto A. Evidence for a local endothelin-angiotensin-atrial natriuretic peptide systemin bovine mature follicles in vitro: Effects on steroid hormones and prostaglandin secretion. Biol Reprod. 1999;61:1419-25.

42. Puglisi R, Cambuli C, Capoferri R, Giannino L, Lukaj A, Duchi R, et al. Differential gene expression in cumulus oocyte complexes collected by ovum pick up from repeat breeder and normally fertile Holstein Friesian heifers. Anim Reprod Sci. 2013;141:26-33.
43. Nishimura T, Hattori A, Takahashi K. Relationship between degradation of proteoglycans and weakening of the intramuscular connective tissue during post-mortem ageing of beef. Meat Sci. 1996;42:251-60.

44. Dubost A, Micol D, Picard B, Lethias C, Andueza D, Bauchart D, et al. Structural and biochemical characteristics of bovine intramuscular connective tissue and beef quality. Meat Sci. 2013;95:555-61.

45. Watanabe R, Chano T, Inoue H, Isono T, Koiwai O, Okabe H. Rb1cc1 is critical for myoblast differentiation through $\mathrm{Rb} 1$ regulation. Virchows Arch. 2005;447:643-8.

46. Sun J, Zhang C, Lan X, Lei C, Chen H. Exploring polymorphisms and associations of the bovine MOGAT3 gene with growth traits. Genome. 2012;55:56-62.

47. Dupont-Versteegden EE, Nagarajan R, Beggs ML, Bearden ED, Simpson PM, Peterson CA. Identification of cold-shock protein RBM3 as a possible regulator of skeletal muscle size through expression profiling. Am J Physiol Regul Integr Comp Physiol. 2008;295:R1263-73.

48. Smith LC, Suzuki Jr J, Goff AK, Filion F, Therrien J, Murphy BD, et al. Developmental and epigenetic anomalies in cloned cattle. Reprod Domest Anim. 2012;47:107-14.

49. Wang $M$, Zhang $X$, Kang $L$, Jiang $C$, Jiang Y. Molecular characterization of porcine NECD, SNRPN and UBE3A genes and imprinting status in the skeletal muscle of neonate pigs. Mol Biol Rep. 2012;39:9415-22.

\section{Submit your next manuscript to BioMed Central and take full advantage of:}

- Convenient online submission

- Thorough peer review

- No space constraints or color figure charges

- Immediate publication on acceptance

- Inclusion in PubMed, CAS, Scopus and Google Scholar

- Research which is freely available for redistribution 